\title{
On Combining Pinpoint Test Set Relaxation and Run-Length Codes for Reducing Test Data Volume*
}

\author{
Seiji Kajihara Yasumi Doi \\ Dept. of Computer Sciences and Electronics \\ Kyushu Institute of Technology
}

\author{
Lei Li Krishnendu Chakrabarty \\ Dept. of Electrical and Computer Engineering \\ DukeUniversity
}

\begin{abstract}
This paper presents a pinpoint test set relaxation method for test compression that maximally derives the capability of a run-length encoding technique such as Golomb coding or frequency-directed run-length (FDR) coding. Before encoding a given set of test patterns, we selectively relax some specified bits of the test patterns. By changing a specified bit with value 1 to a don't-care, two consecutive runs of $0 s$ in the test sequence can be concatenated into a longer run of 0 , thereby facilitating run-length coding. This procedure retains the fault coverage of the test set. Since the increase in compression depends on the lengths of the two runs that are concatenated with each bit relaxation, a lookup table, referred to as the gain table, is pre-computed and used during the test set relaxation procedure to maximize the likelihood of increasing the amount of test data compression. The gain table is used to pinpoint the bit positions with value 1, which when relaxed to don't-cares, will yield the most compression. In this way, the given test pattern set is appropriately modified as a preprocessing step before test compression. Experimental results for the ISCAS benchmark circuits show that the proposed method can be used to increase the effectiveness of run-length coding methods for test data compression.
\end{abstract}

\section{Introduction}

Test cost is a major component of the manufacturing cost for system-on-chip (SOC) integrated circuits. In particular, test data volume is a major contributor to SOC test cost. Large test data volumes lead to an increase in testing time and the need for multiple ATE channel reloads, either from workstations across a network or from slow hard disks. As a result, a number of techniques have recently been presented in the literature to reduce test data volume, and

\footnotetext{
*The work of S. Kajihara was supported in part by Monbu Kagakusho Grants-in-Aid for Scientific Research 14780228. The work of L. Li and K. Chakrabarty was supported by the National Science Foundation under grants CCR-9875324 and CCR-0204077, and by a graduate fellowship from the Design Automation Conference.
}

thereby reduce test cost [1-15]. One such technique is test compaction, which relies on ATPG techniques to generate a small test set with maximum fault coverage $[1,2,3]$. Techniques to generate test sets with a close-to-minimum number of patterns have been developed for full-scan sequential circuits [2,3]. For scan-based circuits however, test compaction alone does not provide sufficient reduction in test data volume if the number of scan flip-flops is very large, since test data volume is proportional not only to the number of test vectors but also to the number of scan flip-flops.

Recently, a number of methods have been proposed for reducing test data volume using compression methods. The key idea here is to compress test data using one of several techniques and use a small amount of embedded hardware for on-chip decompression. Test compression methods can be classified in terms of the type of embedded hardware employed for test delivery. For example, when the decompression hardware is based on an LFSR, reseeding is one of the efficient test compression methods $[4,5,6]$. There also exist several methods for compressing test data by encoding test sets using statistical and run-length encoding [711]. The decompression hardware for these methods consists of a sequential circuit implementing a finite state machine. Test compression methods that use a combinational circuit as a decompressor have also been proposed in the literature $[12,13]$. Finally, a number of methods using an embedded processor for software-based decompression have been proposed, e.g. [20].

Test compression is sometimes based on test patterns generated by ATPG. It is known that unspecified values of the test patterns are very useful to derive higher compression ratio. On the other hand, test generation with leaving unspecified values cannot make the size of the test set be smaller because compaction using reverse order fault simulation [16] or double detection [2] might be useless for the unspecified test patterns. Although high compression ratio can be obtained from a larger test set in general, the resulting test data volume from the large test set may still be larger than that from a compacted test set for the same circuit. Thus if we can compress a highly compacted test set efficiently, test data volume can be reduced. 
When a highly compacted test set is generated in which every bit of test patterns is specified as either to 0 or 1 , there exist values in the test set that can be changed to the opposite logic values without losing fault coverage. One can regards such input values as don't-care (X). A method, called maximal compaction, was developed for identifying an $\mathrm{X}$ in a test pattern for a fault [1]. A method for identifying the Xs in the entire test set was proposed in [17]. Since there are many combinations of $\mathrm{X}$ inputs in general, the method finds one including as many $\mathrm{X}$ inputs as possible. It is reported that, even for a highly compacted test set for ISCAS benchmark circuits, approximately half of values are Xs. Since we can treat the Xs of test patterns as well as unspecified values, we can utilize the Xs for test compression.

In this paper, we present a test modification method for test compression that maximally derives the capability of a run-length based encoding such as Golomb encoding or FDR encoding. Before encoding given test patterns, we efficiently change values of some bits of the test patterns using a technique of don't-care identification [18]. This procedure retains the fault coverage of the test set. The bit values to be changed are selected so that two runs in the test patterns are concatenated into one longer run. Since a gain with each concatenation is different depending on the run-length of prefix and tail for the changed bit, we prepare and refer a gain table that gives the number of bits reduced by encoding after concatenation of two runs. According to the gain table, we pinpoint bit positions that produce larger gain for test modification, and apply the don't-care identification such that Xs on the bits are found maximally. The procedure of test modification is iteratively applied to the test patterns. As a result, the given test patterns are modified optimally for test compression. Experimental results for ISCAS benchmark circuits show that the proposed method can compress test sets efficiently.

This paper is organized as follows. In Section 2, we explain techniques used in the proposed method. In Section 3 , we explain the proposed method. In Section 4, we present experimental results for ISCAS benchmark circuits and finally conclude this paper in Section 5.

\section{Preliminaries}

In this section, we explain techniques used in our work.

\subsection{Run-length based encoding}

As methods for encoding test patterns, FDR (Frequency Directed Run-length) encoding [8] and Golomb encoding [7] is well-known. These methods compress test data by encoding successive 0 s in the test patterns with a codeword. In this paper, each set of test patterns before and after encoding are denoted by $T_{D}$ and $T_{E}$, respectively.

Table 1(a) and Table 1(b) show an example of code assignments with FDR coding and Golomb coding with group
Table 1 Example of codeword assignment

(a) FDR coding

\begin{tabular}{|c|c|c|c|c|}
\hline Group & run-length & Prefix & Tail & Codeword \\
\hline \multirow[t]{2}{*}{ A1 } & 0 & \multirow[t]{2}{*}{0} & \multirow{2}{*}{$\begin{array}{l}0 \\
1\end{array}$} & 00 \\
\hline & 1 & & & 01 \\
\hline \multirow[t]{4}{*}{$\mathrm{A} 2$} & 2 & \multirow[t]{4}{*}{10} & \multirow{4}{*}{$\begin{array}{l}00 \\
01 \\
10 \\
11\end{array}$} & 1000 \\
\hline & 3 & & & 1001 \\
\hline & 4 & & & 1010 \\
\hline & 5 & & & 1011 \\
\hline \multirow[t]{8}{*}{ A3 } & 6 & \multirow[t]{8}{*}{110} & \multirow{8}{*}{$\begin{array}{l}000 \\
001 \\
010 \\
011 \\
100 \\
101 \\
110 \\
111\end{array}$} & 110000 \\
\hline & 7 & & & 110001 \\
\hline & 8 & & & 110010 \\
\hline & 9 & & & 110011 \\
\hline & 10 & & & 110100 \\
\hline & 11 & & & 110101 \\
\hline & 12 & & & 110110 \\
\hline & 13 & & & 110111 \\
\hline
\end{tabular}

(b) Golomb coding

\begin{tabular}{|c|c|c|c|c|}
\hline Group & run-length & Prefix & Tail & Codeword \\
\hline \multirow[t]{4}{*}{ A1 } & 0 & \multirow[t]{4}{*}{0} & \multirow{4}{*}{$\begin{array}{l}00 \\
01 \\
10 \\
11\end{array}$} & 000 \\
\hline & 1 & & & 001 \\
\hline & 2 & & & 010 \\
\hline & 3 & & & 011 \\
\hline \multirow[t]{4}{*}{$\mathrm{A} 2$} & 4 & \multirow[t]{4}{*}{10} & \multirow{4}{*}{$\begin{array}{l}00 \\
01 \\
10 \\
11\end{array}$} & 1000 \\
\hline & 5 & & & 1001 \\
\hline & 6 & & & 1010 \\
\hline & 7 & & & 1011 \\
\hline \multirow[t]{4}{*}{ A3 } & 8 & \multirow[t]{4}{*}{110} & \multirow{4}{*}{$\begin{array}{l}00 \\
01 \\
10 \\
11\end{array}$} & 11000 \\
\hline & 9 & & & 11001 \\
\hline & 10 & & & 11010 \\
\hline & 11 & & & 11011 \\
\hline
\end{tabular}

$t_{1}: 00100$
$t_{2}: 10101$
$t_{3}: 00011$

(a) Given tests

\section{$T D: 001001010100011$ \\ TE: 100010000101100100}

(b) Test stream

Fig. 1 Example of FDR coding $t_{1}^{\prime}: \times 0100$

$t_{2}^{\prime}: 101 \times 1$

$t_{3}$ ':000x1

$\begin{array}{ll}\text { (a) A mask set } & \text { (b) Tests with Xs }\end{array}$

Fig. 2 Don't care identification

size 4, respectively. When a test set in Fig. 1(a) is a given, it is compressed with the FDR encoding as shown in Fig. 1(b). Note that we treat test patterns in the given test set as one stream by concatenating all the test patterns in serial.

The run-length based encoding produces shorter codes, when there are more $0 \mathrm{~s}$ in the test patterns and the length of each run is longer. Therefore the more unspecified values the test set includes, the lesser the test data 
volume is potentially.

\subsection{Don't-care identification}

Test patterns of a test set generated do not have unspecified values generally because they are specified by random fill or static/dynamic test compaction [19] for the detection of faults other than the target fault. However, some primary input values may be changed to opposite logic values without losing fault coverage. One can regard such input values as don't-cares (Xs). A method for identifying Xs of a generated test set has been proposed in [17]. The method in [17] determines as many Xs as possible in the test patterns of the given test set.

To Xs identified, logic values can be reassigned for the purpose of reducing test data volume. If a reassigned value is the same as the original value, the $\mathrm{X}$ is meaningless. Generally the test set with Xs is not uniquely determined, and there are many possible combinations of bit position of the Xs. Desired bit position of the Xs depends on the purpose of the reassignments to the Xs, while the Xs are typically used for test compaction/compression or test power reduction. If it is known that some bits do not have to be changed to Xs, Xs on the other bits can be increased.

A method for placing the Xs on a maximal number of specific bits of a test set has been proposed in [18]. The method allows a user to specify bits for the placement of Xs for each one of the given test vectors. The specific set of bits that should be maximally changed to Xs is specified using a pattern set given together with the given test set. We call the pattern set a mask pattern set, and in short a mask set. Each mask pattern is associated with a test pattern. An example of a mask set for test set $T$ in Fig. 1(a) is shown in Fig. 2(a) where ' $x$ ' means a bit to be changed to an $\mathrm{X}$, and ' $f$ ' means a fixed bit, i.e., an $\mathrm{X}$ on the bit is not needed. An example of a possible test set is shown in Fig. 2(b).

\section{Proposed method}

\subsection{Motivation}

It can be easily imagined that the $\mathrm{X}$ identification technique is suitable for test compression of the given test patterns. Especially, when we can specify bit positions to be changed to an $\mathrm{X}$, the test patterns would be modified so as to derive the effect of test compression maximally. For the run-length encoding, since we need more 0s in the test set, we should prepare a mask set such that bit positions with 1 in the given test set are set to ' $x$ ', and the others are set to ' $f$ '.

Suppose that the test set in Fig. 1(a) is compressed. The mask set for the test set would be prepared as shown in Fig. 3(a). For the original test stream $T D_{0}$, we consider two possible streams, $T D_{1}$ and $T D_{2}$ in Fig. 3(b), which may be obtained from possible test sets with Xs. When we compress

$$
\begin{array}{ll}
m_{1}: \text { ffxff } & T D_{0}: 001001010100011 \\
m_{2}: \operatorname{xxfx} & T D_{1}: 00 \times 0010101000 \times 1 \\
m_{3}: \text { fffxx } & T D_{2}: 0010010 \times 01000 x 1
\end{array}
$$

(a) A mask set

(b) Test stream

Fig. 3 Example of FDR coding

$$
\begin{aligned}
& T E_{0}: 100010000101100100 \\
& T E_{1}: 101101011010 \\
& T E_{2}: 1000100010011010
\end{aligned}
$$

Fig. 4 Compressed test streams after assigning 0 to $\mathrm{Xs}$

$T D_{1}$ with FDR encoding according to Table 1(a), compressed test data $T E_{1}$ as shown in Fig. 4 is obtained because by assigning value 0 on the $\mathrm{Xs}$ in $T E_{1}$. The gain obtained by modifying $T D_{0}$ to $T D_{1}$, which is calculated by $\left|T E_{0}\right|-\left|T E_{1}\right|$, is 4 bits, where $\left|T E_{i}\right|$ means the number of bits of $T E_{i}$. On the other hand, from $T D_{2}, T E_{2}$ is obtained and no gain is obtained by the modification. Although the number of Xs in $T D_{1}$ and $T D_{2}$ is the same, the gains are different. It is observed that there is a bit in the original test set that produces larger gain by changing it to an $\mathrm{X}$, while there is a bit in the original test set that does not produce large gain by changing it to an $\mathrm{X}$.

\subsection{Gain table}

By changing a bit value, two runs in the test patterns are concatenated into one longer run. As shown in the above example, a gain with each concatenation is different depending on the run-length of 0 s in prefix and tail for the changed bit. In the case of $T D_{1}$ in Fig. 3(b), a run with length 2 was concatenated with another run with length 2 . As a result, a run with length 5 was created by assigning 0 to the X. From the codeword in Table 1(a), since both codes of the run with length 2 and the run with length 5 consist of 4 bits, the gain by changing the bit was 4 bits. On the other hand, in the case of $T D_{2}$ in Fig. 3(b), two runs with length 1 were concatenated. But no gain was obtained because two runs with length 1 make a 2-bit code twice and the concatenated run with length 3 makes a 4-bit code.

Based on the table for code assignments in Table 1, we can compute the gain for each bit whose value is 1 in the given test streams when the bit value is changed to 0 . The gain is independent of generation of the test set and just computed from the prefix run-length and the tail run-length for the bit with value 1 statically. Hence we prepare and refer a 2-dimensional table that gives the number of bits reduced by encoding after concatenation of two runs. We call it $a$ gain table. The gain table for FDR coding is given in Table. 2 . The line number and the column number indicate the 
Table 2 Gain table for FDR coding

\begin{tabular}{|r|llllllllllllllll|}
\hline & 0 & 1 & 2 & 3 & 4 & 5 & 6 & 7 & 8 & 9 & 10 & 11 & 12 & 13 & 14 & 15 \\
\hline 0 & 2 & 0 & 2 & 2 & 2 & 0 & 2 & 2 & 2 & 2 & 2 & 2 & 2 & 0 & 2 & 2 \\
1 & 0 & 0 & 2 & 2 & 0 & 0 & 2 & 2 & 2 & 2 & 2 & 2 & 0 & 0 & 2 & 2 \\
2 & 2 & 2 & 4 & 2 & 2 & 2 & 4 & 4 & 4 & 4 & 4 & 2 & 2 & 2 & 4 & 4 \\
3 & 2 & 2 & 2 & 2 & 2 & 2 & 4 & 4 & 4 & 4 & 2 & 2 & 2 & 2 & 4 & 4 \\
4 & 2 & 0 & 2 & 2 & 2 & 2 & 4 & 4 & 4 & 2 & 2 & 2 & 2 & 2 & 4 & 4 \\
5 & 0 & 0 & 2 & 2 & 2 & 2 & 4 & 4 & 2 & 2 & 2 & 2 & 2 & 2 & 4 & 4 \\
6 & 2 & 2 & 4 & 4 & 4 & 4 & 6 & 4 & 4 & 4 & 4 & 4 & 4 & 4 & 6 & 6 \\
7 & 2 & 2 & 4 & 4 & 4 & 4 & 4 & 4 & 4 & 4 & 4 & 4 & 4 & 4 & 6 & 6 \\
8 & 2 & 2 & 4 & 4 & 4 & 2 & 4 & 4 & 4 & 4 & 4 & 4 & 4 & 4 & 6 & 6 \\
9 & 2 & 2 & 4 & 4 & 2 & 2 & 4 & 4 & 4 & 4 & 4 & 4 & 4 & 4 & 6 & 6 \\
10 & 2 & 2 & 4 & 2 & 2 & 2 & 4 & 4 & 4 & 4 & 4 & 4 & 4 & 4 & 6 & 6 \\
11 & 2 & 2 & 2 & 2 & 2 & 2 & 4 & 4 & 4 & 4 & 4 & 4 & 4 & 4 & 6 & 6 \\
12 & 2 & 0 & 2 & 2 & 2 & 2 & 4 & 4 & 4 & 4 & 4 & 4 & 4 & 4 & 6 & 6 \\
13 & 0 & 0 & 2 & 2 & 2 & 2 & 4 & 4 & 4 & 4 & 4 & 4 & 4 & 4 & 6 & 6 \\
14 & 2 & 2 & 4 & 4 & 4 & 4 & 6 & 6 & 6 & 6 & 6 & 6 & 6 & 6 & 8 & 6 \\
15 & 2 & 2 & 4 & 4 & 4 & 4 & 6 & 6 & 6 & 6 & 6 & 6 & 6 & 6 & 6 & 6 \\
\hline
\end{tabular}

length of the prefix bit and the tail bit, respectively. When creating a mask set, according to the gain table we pinpoint bit positions with large gain. Thus we can modify the given test patterns so as to be compressed maximally.

\subsection{Pinpoint bit modification}

In order to avoid identifying Xs on bits with small gain, we define a threshold of gain, $g_{\min }$. We check the gain of each bit with value 1 according to the gain table and create a mask set so that if the gain of a bit is grater than or equal to $g_{\text {min }}$, then put ' $x$ ' on the bit, otherwise put ' $f$ '. We initially set $g_{\min }$ to 8 , and we iterate a procedure to identify Xs and to assign 1 to the $\mathrm{X}$ as long as $\mathrm{Xs}$ are found. If the test set cannot be modified for the given, we decrease $g_{\min }$ and continue the procedure.

We give an outline of the procedure below.

Step 0: Set a given test set to $T$, and set $g_{\text {min }}$ to 8 .

Step 1: From test set $T$ and the gain table, create a mask set such that bits whose gain is grater than or equal to $g_{\min }$ are set to ' $x$ ', and the other bits are set to ' $f$ '.

Step 2: identify Xs on bits in $T$ specified by the mask set.

Step 3: For each $\mathrm{X}$ identified, if the gain by assigning 0 to the $\mathrm{X}$ is still greater than or equal to $g_{\min }$, assign 0 to the $\mathrm{X}$. Otherwise, reassign 1 to the X. Let $T$ ' be the resulting test set.

Step 4: If $T=T^{\prime}$, then set $g_{\text {min }}$ to $g_{\text {min }}-2$. If $g_{\text {min }}<0$, then stop. Step 5: Set $T$ ' to $T$, and go to Step 1 .

Although the don't-care identification is performed based on the test set and the mask set, we don't assign 0 not to all Xs found. This is because, after a value of a bit

$$
\begin{array}{r}
T D_{0}: 001001010100011 \\
\text { gain: } 004002000200022 \\
\text { mask set_0:ffxffffffffffff } \\
T D_{0}^{\prime}: 00 \mathrm{x} 001010100011 \\
T D_{1}: 000001010100011 \\
\text { gain: } 000000000200022 \\
\text { mask set_l:fffffffffxfffxx } \\
T D_{1},: 000001010 \mathrm{f} 000 \mathrm{x} \\
T D_{2}: 000001010000011 \\
\text { gain: } 000000000000002 \\
\ldots \ldots \ldots
\end{array}
$$

Fig.5 An example of pinpoint test relaxation

was changed to 0 , the gains on the adjacent bit with " 1 " may vary. The gain by two bit change is not equal to the sum of the gains by individual bit change. This procedure is based on a greedy algorithm in order to save computing time, i.e., we try to change the bits with larger gain obtained with individual bit change. Hence the optimum solution on the modified test patterns is not guaranteed.

In Fig. 5 we illustrate an example of the procedure. Example: We assume that a test set $T D_{0}$ is given. The gain of each bit is calculated as shown in the second line. As there is no bit whose gain is greater than 4 , we start explanation from the case of $g_{\text {min }}=4$. The mask set for $T D_{0}$ is created in mask set_0. Suppose that a test set $T D_{0}$ ' is obtained from $T D_{0}$ and mask set_O. Then, value 0 is assigned to the $\mathrm{X}$ in $T D_{0}$, , and resultant test set becomes $T D_{1}$. Depending on the gain of each bit in $T D_{1}$, mask set_l is created, where $g_{\text {min }}=2$. We suppose that two Xs in $T D_{1}$ are identified as shown in $T D_{1}$ '. While we assign 0 to the left $\mathrm{X}$, we don't assign 0 to the right $\mathrm{X}$ because the gain is reduced to 0 that is less than $g_{\min }$ after the assignment to the left X. The procedure would still continue after obtaining $T D_{2}$, until the test set cannot be modified. We omit the explanation here.

\section{Experimental results}

We implemented the proposed method on a PC (PentiumIII 1.80GHz, 256MB) using C programming language, and applied it for fully scanned ISCAS' 89 benchmark circuits. As the initial test sets, we prepared highly compacted test sets [2], and used a 200x200 matrix as the gain tables. Table 3 shows experimental results for FDR coding and Golomb coding with $m=4$. The second column and the third column list the number of test patterns in each test set and the number of bits in each test pattern to be compressed. We refer to volume of the original test data as $T D$, and volume of the compressed test data as TE. The columns headed "FDR" and "Golomb" present the number of 
bits compressed with FDR coding and Golomb coding, respectively. Although the proposed method is available for Golomb coding, it brings better results for FDR coding.

We analyzed the procedure and data compressed with FDR coding. Table 4 presents some data for discussion. The columns headed "\#1s" and "\#1 to0s" gives the number of bits whose value is 1 in the original test set, and the number of bits whose value is changed from 1 to 0 by the proposed method. The following five columns present the number of iterations for each $g_{\min }$ in the procedure. The last column gives run time. The proposed method could changes approximately $75 \%$ of $1 \mathrm{~s}$ of the given test set to 0 . As the most of the changes were done in $g_{\min }=2$, we would consider additional criterion for pinpointing bits in $g_{\text {min }}=2$ as a future work. While the iterations of the procedure were more

Table 3 Compression results with FDR coding and Golomb coding $(m=4)$

\begin{tabular}{|l|r|r|c|c|c|}
\hline circuit & \#tests & \#inputs & $\begin{array}{c}\text { TD } \\
\text { [bits] }\end{array}$ & $\begin{array}{c}\text { FDR } \\
\text { [bits] }\end{array}$ & $\begin{array}{c}\text { Golomb } \\
{[\text { bits }]}\end{array}$ \\
\hline s5378 & 100 & 214 & 21400 & 10490 & 12454 \\
s9234 & 111 & 247 & 27417 & 15454 & 18096 \\
s13207 & 235 & 700 & 164500 & 22840 & 52890 \\
s15850 & 97 & 611 & 59267 & 18466 & 26077 \\
s35932 & 12 & 1763 & 21156 & 18072 & 26609 \\
s38417 & 87 & 1664 & 144768 & 64078 & 77711 \\
s38584 & 114 & 1464 & 166896 & 59262 & 74991 \\
\hline
\end{tabular}

than twenty times, it was done still in practical computing time.

Table 5 gives comparisons of the proposed method with others. The column of "nonpinpoint" shows the results without using the gain table, i.e., the procedure tries to change all $1 \mathrm{~s}$ in the test set to $0 \mathrm{~s}$ without notice. The following columns gives results of EFDR coding [9], variable-length Huffman coding [14], RESPIN++ [15], and XOR network [12]. For many circuits, the proposed method showed the highest performance on compression. For s35932, however, our method could not work very well, because the number of bits with value 1 in the test set was greater than that with value 0 . For such a circuit, we should change value 0 to 1 in the test set, and compress it based on the runs with 1 .

\section{Conclusion}

In this paper, we presented a test modification method for test compression that maximally derives the capability of a run-length based encoding such as FDR encoding. Before encoding given test patterns, we changed values of some bits of the test patterns using the technique of don'tcare identification that retains the fault coverage of the test set in any way. The bit values to be changed were selected such that two runs in the test patterns are concatenated into one longer run. In order to maximize the gain obtained by the concatenation, we prepared and referred a gain table that gives the number of bits reduced by encoding after

Table 4 Detailed Results for FDR coding

\begin{tabular}{|l|c|r|r|r|r|r|r|r|}
\hline & \multirow{2}{*}{$\begin{array}{c}\text { \#1s } \\
\text { circuit }\end{array}$} & \#1to0s & \multicolumn{5}{|c|}{ \#iterations } & time \\
\cline { 4 - 8 } & {$[$ bits] } & $g_{\min }=8$ & $g_{\min }=6$ & $g_{\min }=4$ & $g_{\min }=2$ & $g_{\min }=0$ & [sec] \\
\hline s5378 & 11333 & 8489 & 1 & 2 & 3 & 29 & 3 & 31 \\
s9234 & 15154 & 10727 & 1 & 2 & 4 & 17 & 4 & 90 \\
s13207 & 72141 & 67555 & 3 & 4 & 4 & 133 & 4 & 1184 \\
s15850 & 28748 & 24212 & 1 & 2 & 4 & 49 & 4 & 412 \\
s35932 & 13457 & 5595 & 2 & 2 & 3 & 17 & 7 & 501 \\
s38417 & 94695 & 78147 & 1 & 2 & 4 & 77 & 8 & 2254 \\
s38584 & 67733 & 54357 & 2 & 3 & 6 & 42 & 4 & 1216 \\
\hline
\end{tabular}

Table 5 Comparisson of compression results

\begin{tabular}{|l|c|c|c|c|c|c|}
\hline cirucit & ours & nonpinpoint & $\begin{array}{c}\text { EFDR } \\
{[9]}\end{array}$ & $\begin{array}{c}\text { VIHC } \\
{[14]}\end{array}$ & $\begin{array}{c}\text { RESPIN++ } \\
{[15]}\end{array}$ & $\begin{array}{c}\text { XORnet } \\
{[12]}\end{array}$ \\
\hline s5378 & $\underline{10490}$ & 10572 & 11419 & 11516 & 17332 & N/A \\
s9234 & $\underline{\underline{15454}}$ & 15846 & 21250 & 17736 & 17198 & N/A \\
s13207 & $\underline{\underline{22840}}$ & 24736 & 29992 & 27737 & 26004 & 25344 \\
s15850 & $\underline{18466}$ & 19906 & 24643 & 30271 & 32226 & 22784 \\
s35932 & 18072 & 17550 & $\underline{5554}$ & 9458 & N/A & 7128 \\
s38417 & $\underline{64078}$ & 66020 & 64962 & 74938 & 89132 & 89356 \\
s38584 & $\underline{59262}$ & 61960 & 73853 & 85674 & 63232 & $\underline{38976}$ \\
\hline
\end{tabular}


concatenation of two runs. Then, we pinpointed bit positions, and apply the don't-care identification. The procedure of test modification was iteratively applied to the test patterns. Experimental results for ISCAS benchmark circuits showed that the proposed method could compress test sets efficiently.

\section{Reference}

[1] I. Pomeranz, L. N. Reddy and S. M. Reddy, "COMPACTEST: A Method to Generate Compact test Sets for Combinational Circuits," IEEE Trans. CAD, pp. 1040-1049, Jul. 1993.

[2] S. Kajihara, I. Pomeranz, K. Kinoshita and S. M. Reddy, "Cost-Effective Generation of Minimal Test Sets for Stuckat Faults in Combinational Logic Circuits," IEEE Trans. CAD, pp.1496-1504, Dec. 1995.

[3] I. Hamzaoglu and J. H. Patel, "Test Set Compaction Algorithms for Combinational Circuits," Proc. Int'l Test Conf, pp. 283-289, Oct. 1998

[4] S. Hellebrand, J. Rajski, S. Tarnick, S. Venkataraman and B. Courtois, "Built-in Test for Circuits with Scan Based on Reseeding of Multiple-polynomial Linear Feedback Shift Registers," IEEE Trans. Comp., pp. 223-233, Feb. 1995.

[5] S. Hellebrand, H.-G. Liang, H. -J. Wunderlich, "A mixed-mode BIST scheme based on reseeding of folding counters," Int'l Test Conf., pp.778-784, Oct. 2000.

[6] C. V. Krishna, A. Jas, and N. A. Touba, "Test Vector Encoding Using Partial LFSR Reseeding," International Test Conf., pp. 885-893, Oct. 2001.

[7] A. Chandra and K. Chakrabarty, "Test Data Compression for System-on-a-Chip Using Golomb Codes," VLSI Test Symposium, pp. 113-120, April 2000.

[8] A. Chandra and K. Chakrabarty, "Frequency-directed Runlength (FDR) Codes with Application to System-on-a-chip Test Data Compression,” Proc. VLSI Test Symp., pp. 42-47, April 2001.

[9] A. El-Maleh, R. Al-Abaji, "Extended Frequency-directed Runlength Codes with Improved Application to System-on-aChip Test Data Compression," Int'l Conf. Electronics, Circuits and Systems, pp. 449-452, 2002.
[10] A. Jas, J. Ghosh-Dastidar, and N. A. Tuba, "Scan Vector Compression/Decompression Using Statistical Coding," VLSI Test Symposium, pp. 114-120, April 1999.

[11] H. Ichihara, K. Kinoshita, I. Pomeranz, S. M. Reddy, “Test Transformation to Improve Compaction by Statistical Encoding," International Conf. on VLSI Design, pp.294-299, Jan. 2000.

[12] I. Bayraktaroglu and A. Orailoglu, "Test Volume and Application Time Reduction Through Scan Chain Concealment," Design Automation Conference, pp.151-155, June 2001.

[13] S. M. Reddy, K. Miyase, S. Kajihara and I. Pomeranz, "On Test Data Volume Reduction for Multiple Scan Chain Designs," VLSI Test Symposium, pp. 103-108, April 2002.

[14] P. T. Gonciari, B. Al-Hashimi, N. Nicolici, "Improving compression ratio, area overhead, and test application time for system-on-a-chip test data compression/decompression," Design Automation and Test in Europe Conf., pp. 604-611, 2002.

[15] L. Schafer, R. Dorsch, H. -J. Wunderlich, "RESPIN++ Deterministic Embedded Test," European Test Workshop, pp.37-44, May 2002.

[16] M. Schulz, E. Trischler, and T. Sarfert, "SOCRATES: A Highly Efficient Automatic Test Pattern Generation System,” IEEE Trans. on CAD., pp. 126-137, Jan. 1988.

[17] S. Kajihara, and K. Miyase, “On Identifying Don't Care Inputs of Test Patterns for Combinational Circuits," Proc. Int'l Conf. on Computer Aided Design, pp. 364-369, Nov. 2001.

[18] K. Miyase, S. Kajihara, I. Pomeranz and S. M. Reddy, "Don't care identification on specific bits of test patterns," 2002 International Conference on Computer Design, pp. 194199, Sep. 2002.

[19] P. Goel, and B. C. Rosales, "Test Generation and Dynamic Compaction of Tests," Digest of Papers 1979 Test Conf., pp. 189-192, Oct. 1979

[20] A. Jas and N. A. Touba, "Using an embedded processor for efficient deterministic testing of system-on-achip," Int.' $l$ Conf. on Computer Design, pp.418-423, 1999. 